\title{
Operation drawings of Dame Barbara Hepworth
}

\author{
PAUL BOWNESS
}

The work of Barbara Hepworth (1903-75), like that of Britain's other major sculptor, Henry Moore, includes an important number of paintings and drawings. Among these is a series of 61 operation studies made between 1947 and 1949, which are unique in their treatment of medical (rather than anatomical) subjects. They have received little critical attention because they stand curiously apart from her development as an abstract sculptor. As an artist's view of the operating theatre, however, they offer considerable insight to those of us in the medical profession.

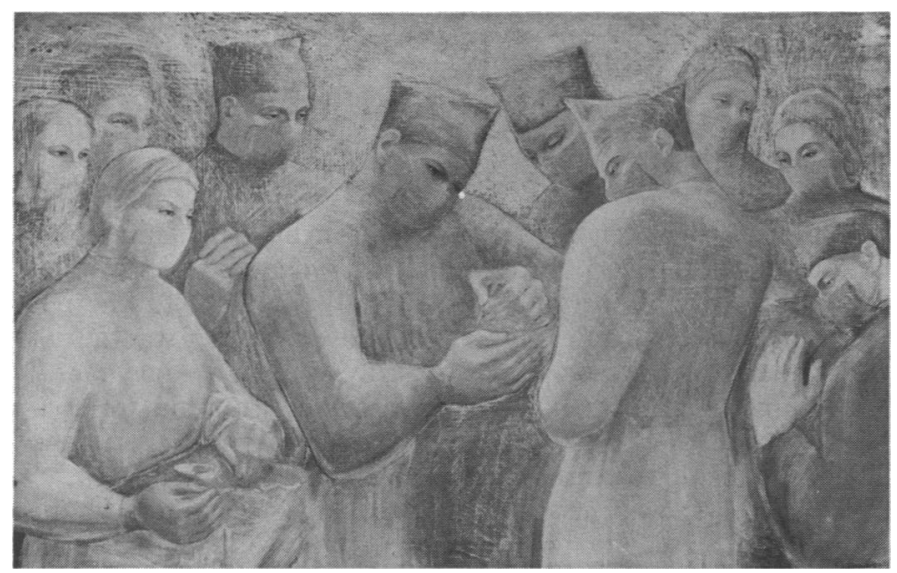

FIG 1-“Concourse No 2," 1948. Cil and pencil on wood panel. $24 " \times 48$ ". Collection: the Royal College of Surgeons of London.

Before turning to the pictures, I will first describe how she came to make them. During the second world war Hepworth, who had been one of the pioneers of British abstract sculpture in the 1930s, was forced by lack of space and materials to turn to drawing. ${ }^{1}$ These abstract compositions, exploring the tension and space within crystal like forms, were further pursued after the war. Hepworth's energy was such that, as well as resuming her sculpture, she also produced two major series of figure and operation drawings.

The operation series stems from her friendship with Norman Capener, the orthopaedic surgeon who had operated on her daughter, Sarah, for osteomyelitis. She was invited to watch operations at the Princess Elizabeth Orthopaedic Hospital in Exeter and later at the National Orthopaedic Hospital and the London Clinic. She was allowed a pen and sterile pad to make notes, but the drawings were done largely from memory immediately afterwards. ${ }^{2}$ She describes her first experience: "When I entered the operating theatre I became completely absorbed by two things: first, the extraordinary beauty of purpose and coordination between human beings all dedicated to the saving of life, and the way that unity of idea and purpose dictated a perfection of concentration, movement, and gesture; and secondly by the way this special grace (grace of mind and body) induced a spontaneous space composition, an articulated and animated kind of abstract sculpture very close to what I had been seeking in my own work."

St Mary's Hospital Medical School, London W2 1NY

PAUL BOWNESS, BA, medical student

Correspondence to: 91 Castelnau, London SW13 9EL.
Thus Hepworth saw for the first time large groups of people working in harmony in what she considered to be an ideal environment. This is reflected in her operation drawings-largely multifigure compositions - where we are made conscious of the unity of the group (fig 1). Before this period her sculpture and drawing had concentrated almost exclusively on single or two related forms. But after her operation series (and contemporary figure drawings of a single nude drawn from different angles) she went on to make multiform abstract sculptures.

\section{Concentration on eyes and hands}

The operation drawings concentrate on the participants' eyes and hands. Hepworth uses the homogeneity of theatre dress to veil figure (and gender); and a green wash to blend dress and background. Looking at the drawing, we see first the eyes, which

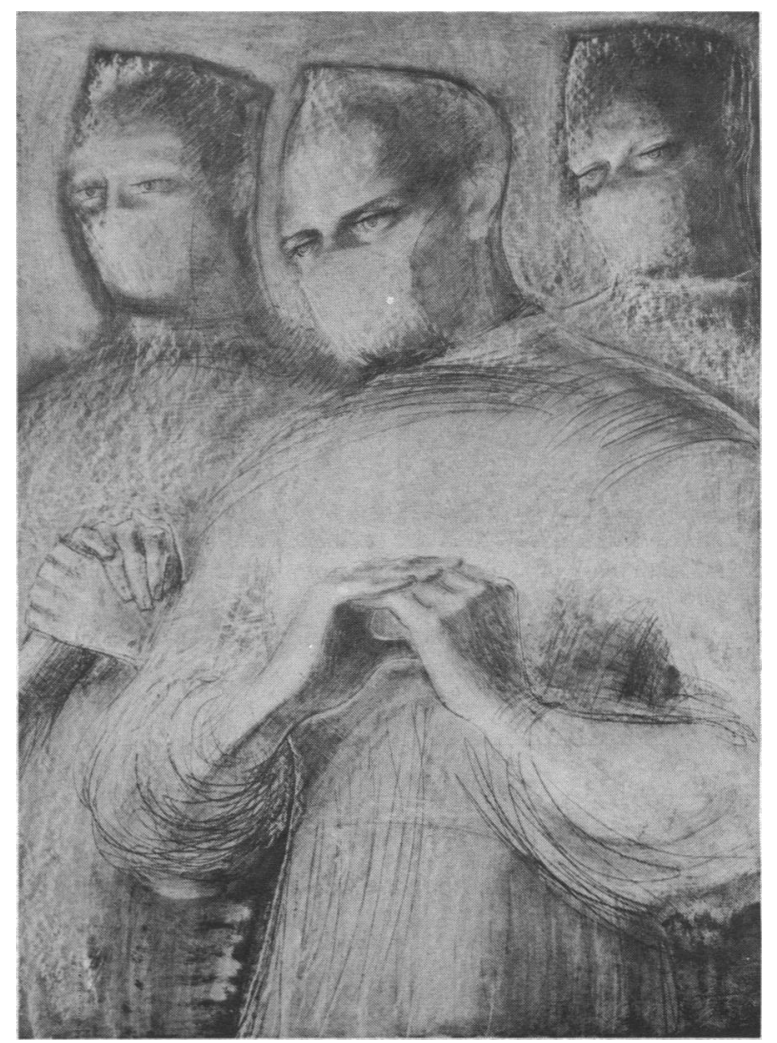

FIG 2-"Skiagram," 1949. Oil and pencil. 211/2" $\times 14^{1 / 2 "}$ ". Collection: Sir William Worsley.

repeatedly direct our attention to the hands. The hands have their own independence-either poised holding instruments or held sterile before use-in a pose that the artist found prayer like and peaceful (fig 2). The combination of precision and strength needed for orthopaedic operations must have seemed remarkably akin to her own expertise in carving marble, especially when hammer and chisel were used.

Hepworth was especially fascinated by hand operations. "The anatomy of the unconscious hand, exposed and manipulated by the 
conscious hand with the scalpel, expresses vividly the creative inspiration of superb coordination in contrast to the unconscious mechanism." "Nevertheless, only five of the 61 drawings are of arm or hand operations. The majority (36) are general scenes; figures in theatre, standing, putting on gloves. She also made four drawings of outpatient clinics, and several depicting specific operations, which represent important documents in the history of orthopaedic surgery. The "Fenestration of the ear" series (1948) shows Garnett Passe at various stages of an operation that he had pioneered in Britain. ${ }^{4}$ The fourth in the series of six is illustrated here (fig 3 ).

This major body of work is especially exciting to all of us who have helped at operations. Barbara Hepworth depicts with an aesthetic eye the arrangement of the operating team, its dress, posture, and action; things that we are trained to see with a professional eye.

\section{References}

1 Hammacher AM. Barbara Hepworth. London: Thames and Hudson, 1968:45-86.

2 Science Museum Pictorial Collection. Sketch book. Science museum inventory. No 1977-500. London.

3 Bowness A. Barbara Hepworth: drawings from a sculptor's landscape. London: Cory, Adams, and Mackay, 1956:22.

4 Morphet R. In: The Tate Gallery 1976-78, illustrated catalogue of acquisitions. London: Tate Gallery Publications, 1979:80-4.

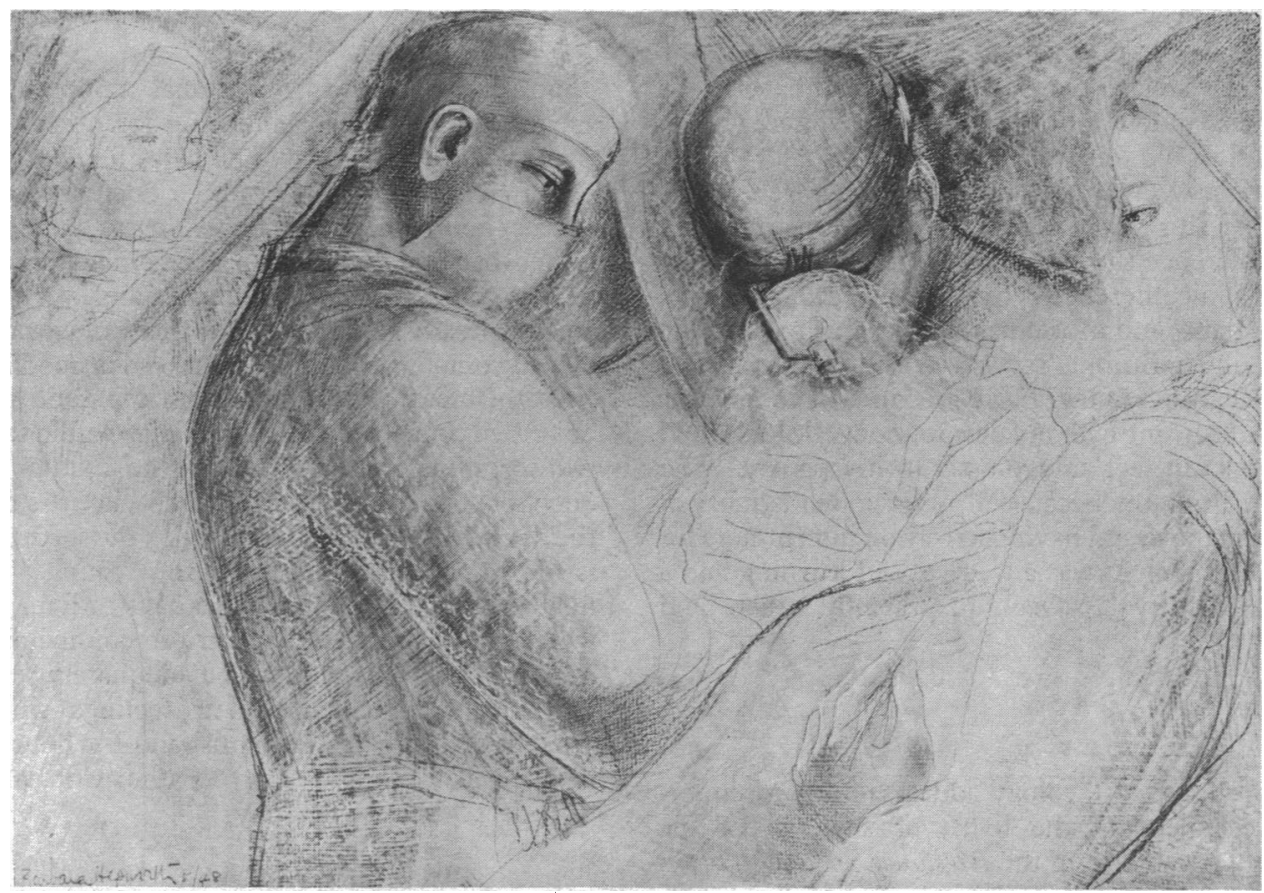

FIG 3- "Fenestration of the Ear No 4. 'The Microscope"' 1948. Oil and pencil. Collection: Leeds City Art Gallery. 